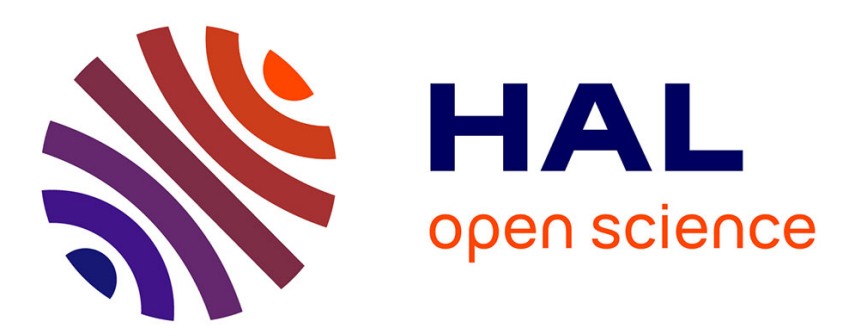

\title{
On finite time resonance entrainment in multi-DOF systems
}

Denis Efimov, Alexander Fradkov, Tetsuya Iwasaki

\section{To cite this version:}

Denis Efimov, Alexander Fradkov, Tetsuya Iwasaki. On finite time resonance entrainment in multiDOF systems. ACC 2012, Jun 2012, Montreal, Canada. hal-00678974

\section{HAL Id: hal-00678974 \\ https://hal.inria.fr/hal-00678974}

Submitted on 14 Mar 2012

HAL is a multi-disciplinary open access archive for the deposit and dissemination of scientific research documents, whether they are published or not. The documents may come from teaching and research institutions in France or abroad, or from public or private research centers.
L'archive ouverte pluridisciplinaire HAL, est destinée au dépôt et à la diffusion de documents scientifiques de niveau recherche, publiés ou non, émanant des établissements d'enseignement et de recherche français ou étrangers, des laboratoires publics ou privés. 


\title{
On finite time resonance entrainment in multi-DOF systems
}

\author{
Denis Efimov, Alexander Fradkov, Tetsuya Iwasaki
}

\begin{abstract}
The mechanism of entrainment to natural oscillations in a class of (bio)mechanical systems described by linear models is investigated. A nonlinear control strategy (based on the speed gradient control algorithm) is analyzed providing the system oscillation in resonance mode with a natural frequency. It ensures an energy-optimal entrainment performance robustly against perturbations in system parameters in a finite time. The obtained controller equations can be interpreted as equations of a dynamical neural network. Assuming that the resetting mechanism in animals is selected to ensure a suboptimal entrainment performance, the neural network interpretation may be helpful for understanding the entrainment phenomenon in nature. Efficiency of the proposed entrainment solutions is demonstrated by examples of computer simulation.
\end{abstract}

Index Terms - entrainment, nonlinear control, oscillations

\section{INTRODUCTION}

$\mathbf{T}$ HE robotic or animal locomotion is a periodical movement synchronized with the environment influence. To minimize the energy consumption during the movement, the body oscillations have to be performed in a resonance mode. For example, the period of walking can be related to the natural frequency of legs as a pendulum [15]. This fact is of great importance for analysis of the locomotion phenomenon in animals and for robotic systems design as well. In this way a fundamental question arises: how to design a feedback controller ensuring reliable oscillations in (or near) a resonance mode?

An answer to this question is based on the fact that rhythmic animal motions are controlled by neural circuits called the Central Pattern Generator (CPG) [2], [14], [16]. CPGs are composed by neural networks generating sustained oscillations and defining the locomotion rhythm. Such a control mechanism of oscillations can be related with the entrainment phenomenon, when one oscillator (CPG) entrains to the frequency and phase of rhythmic movement in another oscillator (in a series or feedback connection). This feedback mechanism is now used in robotics and mechanical systems to regulate locomotion [10], [11]. The conditions of

The first author is with Non-A project at INRIA - LNE, Parc Scientifique de la Haute Borne 40, avenue Halley Bât.A, Park Plaza 59650 Villeneuve d'Ascq, France; e-mail: Denis.Efimov@inria.fr.

The second author is with the Institute for Problems of Mechanical Engineering, 61, Bolshoy av., V.O. , St-Petersburg, 199178 Russia, St.Petersburg State University and National Research University of Information Technology, Mechanics and Optics; e-mail: fradkov@mail.ru.

The third author is with the Department of Mechanical and Aerospace Engineering, University of California, Los Angeles, CA 99095; e-mail: tiwasaki@ucla.edu. resonance regime appearance are analyzed using (multivariable) Harmonic Balance (HB), and hence are approximate in nature.

The goal of this work is to make the next step and develop control methods that achieve resonance entrainment exactly. We would like to propose control algorithms providing a shift of system oscillations to resonance regime with posterior interpretation of the control as a CPG or a neural network. Our underlying idea is that not only the resonance motion is energy-optimal, but its control mechanism may also be a result of a natural optimization. Applying inverse interpretation, we use the speed gradient principle [9] to arrange the type of behavior that was called feedback resonance in [7], [8]. The control equations form a neural network structure similar to [10], [11].

The main results present an energy-optimal control law for a class of multiple degree-of-freedom (DOF) mechanical systems.

Preliminary results are obtained in Section 2 where the resonance controls based on the speed gradient approach from [4], [5], [7], [8] are introduced and applied to a single DOF linear pendulum. Optimality of a form of the speed gradient algorithm for energy excitation in the linear pendulum has been shown in [1]. The problem statement of natural oscillation entrainment in linear mechanical multiDOF systems is presented in Section 3. The control algorithms based on the results from [4], [5] are developed in Section 4. Interpretation of their equations as a dynamical neural network is also given. Efficiency of the obtained control algorithms is demonstrated on examples of computer simulation in Section 5.

\section{Preliminaries}

In this section we will develop basic control strategies by applying the results from [1], [4], [5], [7], [8] to the linear model of a pendulum:

$$
\ddot{x}+\kappa \dot{x}+\omega^{2} x=b u,
$$

where $x \in(-\pi, \pi]$ is the angle of pendulum rotation, $\dot{x} \in \mathbb{R}$ is the angular velocity, $u \in[-U, U]$ is the control, $U \in \mathbb{R}_{+}$ is the maximal control amplitude; $\kappa \in \mathbb{R}_{+}$is the friction coefficient, $\omega \in \mathbb{R}_{+}$is the frequency of oscillations without friction, $b \in \mathbb{R}, b \neq 0$ is the control gain. It is assumed that $|\omega|>\kappa$, in this case the transfer function of the system (1) has poles $\lambda_{1,2}=-0.5 \kappa \pm \omega_{0} i$ with nonzero 
imaginary part $\omega_{0}=\sqrt{\omega^{2}-\kappa^{2} / 4}$, where $\omega_{0}$ is the natural frequency of oscillations for (1). Typically, the oscillations with the frequency $\omega_{0}$ are called resonant for (1). It is worth noting that the Bode magnitude plot of the system (1) reaches its maximum at the frequency $\omega_{1}=\sqrt{\omega^{2}-\kappa^{2} / 2}$ while $\omega \geq \kappa / \sqrt{2}$, and at the zero frequency otherwise (for the variable $\dot{x}$ the resonant frequency is $\omega$ ). The difference between frequencies $\omega, \omega_{0}, \omega_{1}$ is minor if the value of $\kappa$ is sufficiently small, that is the case under consideration.

Due to constraint on the control amplitude, the problem of excitation of the resonance oscillations becomes meaningful from practical point of view. If it is required to ensure oscillations with the maximal amplitude for a given amplitude of the control, then the resonance mode is a solution. The question is how to design such a control $u=u(x, \dot{x})$, $|u| \leq U$ providing the system oscillations with the frequency $\omega_{0}$ or $\omega_{1}$. An additional goal consists in the oscillation phase resetting.

The pendulum (1) energy has form:

$$
E(x, \dot{x})=0.5\left[\dot{x}^{2}+\omega^{2} x^{2}\right], \quad \dot{E}=-\kappa \dot{x}^{2}+b \dot{x} u .
$$

Each fixed value of the energy $E$ corresponds to an oscillation with the angle amplitude $\sqrt{2 E} / \omega$, the presence of friction leads to the energy dissipation. In the paper [1] it is shown that the control

$$
u=U \operatorname{sign}(\dot{x}), \operatorname{sign}(s)= \begin{cases}s /|s| & \text { if } s \neq 0 \\ {[-1,1]} & \text { if } s=0\end{cases}
$$

is the optimal solution of the terminal energy maximization problem. Since the control (2) is piecewise constant, then solutions of the system (1), (2) can be presented as follows:

$$
\begin{aligned}
x(t)= & e^{-\kappa t / 2}\left[\omega_{0}^{-1}\left(\dot{x}(0)+\kappa\left\{x(0)-s_{0}\right\} / 2\right) \sin \left(\omega_{0} t\right)+\right. \\
& \left.+\left\{x(0)-s_{0}\right\} \cos \left(\omega_{0} t\right)\right]+s_{0}, \\
\dot{x}(t)= & e^{-\kappa t / 2}\left[\dot{x}(0) \cos \left(\omega_{0} t\right)-\omega_{0}^{-1}\left(\omega^{2}\left\{x(0)-s_{0}\right\}+\right.\right. \\
& \left.+\kappa \dot{x}(0) / 2) \sin \left(\omega_{0} t\right)\right] \\
s_{0}= & b \omega^{-2} U \operatorname{sign}(\dot{x}(0))
\end{aligned}
$$

for $t \in\left[0, t_{1}\right)$, where

$$
t_{1}=\frac{1}{\omega_{0}} \operatorname{atan}\left(\frac{\omega_{0} \dot{x}(0)}{\omega^{2}\left\{x(0)-s_{0}\right\}+\kappa \dot{x}(0) / 2}\right)
$$

is the instant of time when $\dot{x}\left(t_{1}\right)=0$ (for the first time). Introduce $t_{i}, i \geq 1$ as the sequence of instants satisfying constraints $\dot{x}\left(t_{i}\right)=0$ and $\dot{x}(t) \neq 0$ for $t \neq t_{i}, i \geq 1$. For $t \in\left[t_{i}, t_{i+1}\right), i \geq 1$ we have

$$
\begin{aligned}
x(t)= & e^{-\kappa\left(t-t_{i}\right) / 2}\left\{x\left(t_{i}\right)-s_{i}\right\}\left(\omega_{0}^{-1} \kappa / 2 \sin \left[\omega_{0}\left(t-t_{i}\right)\right]+\right. \\
& \left.+\cos \left[\omega_{0}\left(t-t_{i}\right)\right]\right)+s_{i}, \\
\dot{x}(t)= & -e^{-\kappa\left(t-t_{i}\right) / 2}\left\{x\left(t_{i}\right)-s_{i}\right\} \omega_{0}^{-1} \omega \sin \left[\omega_{0}\left(t-t_{i}\right)\right], \\
s_{i}= & -s_{i-1} .
\end{aligned}
$$

From the expression above we have that $t_{i+1}-t_{i}=\pi / \omega_{0}$, $i \geq 1$. Therefore, the control (2) ensures that the trajectory of system (1) converges to oscillations with the frequency $\omega_{0}$ [4] (the resonance oscillations) in the finite time $t_{1}$. Oscillating at this frequency the closed-loop system trajectories asymptotically converge to the limit cycle path:

$$
\begin{aligned}
x(t)= & \left\{\begin{array}{cc}
\gamma(t), & 0 \leq t \leq \pi / \omega_{0} \\
-\gamma\left(t-\pi / \omega_{0}\right), & \pi / \omega_{0} \leq t \leq 2 \pi / \omega_{0},
\end{array}\right. \\
\gamma(t)= & e^{-\kappa t / 2}\left\{\gamma_{0}-b U / \omega^{2}\right\}\left[\omega_{0}^{-1} \kappa / 2 \sin \left(\omega_{0} t\right)+\right. \\
& \left.+\cos \left(\omega_{0} t\right)\right]+b U / \omega^{2},
\end{aligned}
$$

where

$\gamma_{0}=-b U / \omega^{2}\left(1+e^{-\kappa \pi / \omega_{0}}+2 e^{-\kappa \pi /\left(2 \omega_{0}\right)}\right) /\left(1-e^{-\kappa \pi / \omega_{0}}\right)$

is the oscillation amplitude for the variable $x$ on the limit cycle. Thus we have proven the following result [1], [4], [9].

Lemma 1. For the system (1) with the control (2) the following statements are true:

1) convergence in the finite time $t_{1}$ to the oscillations with the frequency $\omega_{0}$;

2) the asymptotic amplitude of oscillations for the variable $x$ is $\gamma_{0}$.

In the paper [4] an adaptive algorithm for amplitude adjustment in the control (2) is proposed ensuring the required amplitude of oscillations on the limit cycle $\gamma_{0}$ for the case of unknown values of the pendulum (1) parameters. In the paper [3] the same problem (excitation to the resonance mode of the pendulum (1) for unknown values of parameters) is solved using the adaptive observer technique. This control is related with the mechanism of positive rate feedback with saturation discussed in [11].

If the control is chosen in the form

$$
u=-U \operatorname{sign}(\dot{x}),
$$

then it ensures the system (1) stabilization with an optimal performance.

Lemma 2. For the system (1) the control (3) ensures global stability of the set $\mathcal{X}=\{(x, \dot{x}) \in(-\pi, \pi] \times \mathbb{R}:|x| \leq$ $\left.|b| U / \omega^{2}, \dot{x}=0\right\}$ and attractivity of an equilibrium into $\mathcal{X}$, providing the free end terminal optimization of the system energy $E$.

In this work, we will also be interested in the finite time stabilization, that can be realized by the following control law:

$$
u=-\beta \operatorname{sign}(\dot{x})-(\beta+\varepsilon) \operatorname{sign}\left(\kappa \dot{x} / \omega^{2}+x\right),
$$

where $2 \beta+\varepsilon=U$ and $\beta>0, \varepsilon>0$ are the design parameters.

Lemma 3. The control (4) ensures global stabilization of the system (1) in a finite time $t_{0} \geq 0$ :

$$
t_{0} \leq T_{0}=2 \mu^{-1} \sqrt{V\left(\kappa \dot{x}(0) / \omega^{2}+x(0), \dot{x}(0)\right)},
$$


where

$$
\begin{aligned}
V\left(\xi_{1}, \xi_{2}\right) & =0.5\left[0.5 \omega^{2} \xi_{1}^{2}+\xi_{2}^{2}+0.5 \omega^{2}\left(\xi_{1}-\kappa \xi_{2} / \omega^{2}\right)^{2}\right] \\
& +b(\beta+\varepsilon)\left|\xi_{1}\right|
\end{aligned}
$$

and

$$
\begin{aligned}
& \mu=\min \{b(\beta+\varepsilon) \kappa / \omega, b \beta \omega /(\sqrt{0.5} \omega+0.5 \kappa), \\
& \left.0.5 \kappa \omega^{2} / \sqrt{b(\beta+\varepsilon)}, 8 \kappa b \sqrt{b(\beta+\varepsilon)} \varepsilon /\left[3 \sqrt[3]{2} \omega^{2}\right]\right\} .
\end{aligned}
$$

Neither the controls (2), (3) nor (4) regulate the phase of oscillations, and in addition, the resulting oscillations in the resonance mode have non-constant energy $E$. The control presented in [5] makes the regulation of both, the energy $E$ and the phase $\varphi \in[-\pi, \pi)$.

Comparing controls (2), (3), (4), note that the operation of the first two is independent of the system (1) parameters.

Frequently only the angle position $x$ is available for measurements while the controls (2), (3) are based on the velocity $\dot{x}$. As in [3], [4] we will use the following simple filter to evaluate $\dot{x}$.

Lemma 4. Let $|\ddot{x}(t)| \leq \Xi, \Xi \in \mathbb{R}_{+}$for all $t \geq 0$. Then the filter

$$
\dot{q}=-\eta(q+x), \eta>0, \hat{\dot{x}}=\eta(q+x)
$$

provides estimation of $\dot{x(t)}$ with accuracy:

$$
|\hat{\dot{x}}(t)-\dot{x}(t)| \leq|\eta[q(0)+x(0)]-\dot{x}(0)| e^{-\eta t / 2}+2 \sqrt{2} \Xi / \eta \text {. }
$$

This linear filter has asymptotic convergence and a steadystate error bound proportional to $\Xi$, that can be dominated by increasing the value of $\eta$. The following nonlinear filter performs exact differentiation in the noise-free case in a finite time [6].

Lemma 5. Let $|\dot{x}(t)| \leq L_{1} \in \mathbb{R}_{+},|\ddot{x}(t)| \leq L_{2} \in \mathbb{R}_{+}$, for all $t \geq 0$. Then the filter

$$
\begin{aligned}
\dot{\zeta}_{1}= & -\alpha \sqrt{\left|\zeta_{1}-x(t)\right|} \operatorname{sign}\left[\zeta_{1}-x(t)\right]+\zeta_{2}, \\
\dot{\zeta}_{2}= & -\rho \operatorname{sign}\left[\zeta_{1}-x(t)\right]-\chi \operatorname{sign}\left(\zeta_{2}\right)-\zeta_{2}, \\
& \zeta_{1}(0)=x(0), \quad \zeta_{2}(0)=0,
\end{aligned}
$$

where

$$
\begin{gathered}
\chi=0.25 \sqrt[4]{2} L_{1}+\varrho, \rho>L_{1}+L_{2}+3 \chi, \\
\alpha=4\left[\sqrt{2\left(\rho+L_{1}+L_{2}+2 \chi\right)} \chi+\right. \\
\left.\sqrt{\rho+L_{1}+L_{2}+3 \chi}\left(L_{1}+L_{2}+2 \chi\right)\right] / \\
{\left[2 \rho-L_{1}-L_{2}-2 \chi\right]}
\end{gathered}
$$

for some $\varrho \in \mathbb{R}_{+}$, has bounded solutions and $\zeta_{2}(t)=\dot{x}(t)$ for all $t \geq T_{d}$ with $T_{d} \leq L_{1} /\left(0.25 \sqrt[4]{2} L_{1}+\varrho\right)$.

\section{NATURAL OSCILLATION ENTRAINMENT PROBLEM}

Consider the class of mechanical systems with the model

$$
J \ddot{x}+D \dot{x}+S x=B u, y=C x,
$$

where $x \in \mathbb{R}^{n}$ is the vector of generalized coordinates, $u \in$ $\mathbb{R}^{m}$ and $y \in \mathbb{R}^{m}$ are the input and the output respectively.
Assumption 1. The inertia, damping and stiffness matrices satisfy

$$
\begin{aligned}
J= & J^{T}>0, S=S^{T} \geq 0, D=\varepsilon S, \varepsilon>0, \\
& \operatorname{rank}(B)=\operatorname{rank}(C)=m, m=n .
\end{aligned}
$$

Assumption 1 is similar to those used in [10]. Roughly speaking it implies that the system has Rayleigh damping, the vector $x$ is available for measurements and the dimension of the control equals to the number of generalized coordinates. A natural mode of (8) is defined by a pair of the natural frequency $\omega_{i} \in \mathbb{R}_{+}$and the mode shape $\xi_{i} \in \mathbb{R}^{n}$ satisfying $\left(S-\omega_{i}^{2} J\right) \xi_{i}=0, i=\overline{1, n}$ (where the symbol $\overline{1, n}$ is used to denote the sequence of integers $1,2, \ldots, n$ ). For brevity of presentation we will assume that all $\omega_{i}, i=\overline{1, n}$ are different (the approach can be easily extended to the multiple case). We will also assume that the controller is allowed to have centralized information processing.

The problem of natural oscillation entrainment can be formulated now as follows. Design a control $u$ ensuring that the system (8) oscillates at a selected natural frequency $\omega_{k}$, $k \in\{1, \ldots, n\}$.

\section{RESULTS}

This section extends the feedback resonance mechanism in the controls (2), (3) for the single-DOF system to the multiDOF system (8). Our approach is based on the transformation $x=P z$ of the system (8) to the normal mode canonical form:

$$
\ddot{z}+K \dot{z}+\Omega z=R u
$$

where

$$
\begin{aligned}
& \Omega=P^{-1} J^{-1} S P=\operatorname{diag}\left[\omega_{1}^{2}, \ldots, \omega_{n}^{2}\right], \\
& K=P^{-1} J^{-1} D P=\operatorname{diag}\left[\kappa_{1}, \ldots, \kappa_{n}\right], \\
& R=P^{-1} J^{-1} B, \quad \kappa_{i}=\varepsilon \omega_{i}^{2}, \quad i=\overline{1, n},
\end{aligned}
$$

and the columns of the matrix $P$ are composed of the vectors $\xi_{i}$. Under Assumption 1 we may assume that the vector $x=$ $C^{-1} y$ is available for measurements (the matrix $C$ has rank $n$ ). By the same arguments, $\operatorname{since} \operatorname{rank}(B)=n$, the matrix $R$ is nonsingular and introducing an auxiliary control $v=R u$ we may rewrite the system (9) as a sequence of independent systems (1):

$$
\ddot{z}_{i}+\kappa_{i} \dot{z}_{i}+\omega_{i}^{2} z_{i}=v_{i}, i=\overline{1, n} .
$$

Thus to choose $v_{i}, i=\overline{1, n}$ the controls (2), (3), (4) can be used. The $i$-th system in (10) is responsible for the system (8) oscillation on the frequency $\omega_{i}$ or $\omega_{i, 0}=\sqrt{\omega_{i}^{2}-\kappa_{i}^{2} / 4}$. Stabilization of the $i$-th normal mode at the origin (zero energy level) implies elimination of the frequency $\omega_{i}$ from the frequency spectrum of the system (8) oscillation. Thus to solve the problem of natural oscillation entrainment to the frequency $\omega_{k}$, it is necessary to stabilize at the origin all normal modes with $\omega_{i} \neq \omega_{k}$ providing a sufficient level of excitation for the $k$-th mode. For future reference define the set of canceling normal modes $\mathcal{J}=\{1, \ldots, n\} \backslash\{k\}$. 


\section{A. The energy-optimal control}

To excite the $k$-th mode by (2) and attenuate the other modes by (3), we choose

$$
v_{i}=U_{i} \operatorname{sign}\left(\dot{z}_{i}\right), i=\overline{1, n},
$$

where $U_{k}>0$ and $U_{j} \leq 0, j \in \mathcal{J}$ are some constants. Since the variables $\dot{z}_{i}, i=\overline{1, n}$ are not available for measurements, to realize the control (11) we may use the filter (5):

$$
\dot{q}_{i}=-\eta\left(q_{i}+z_{i}\right), \hat{\dot{z}}_{i}=\eta\left(q_{i}+z_{i}\right),
$$

for some $\eta>0$. Then the final expression of the speed gradient control algorithm in this case is as follows:

$$
u=R^{-1} \operatorname{diag}[U] \operatorname{sign}\left(\hat{z}_{i}\right) .
$$

According to lemmas 1, 2 this control ensures a near optimal resonance excitation performance.

This control has a structure similar to the control proposed in [10] (see Section IV-B for details). However, it is hard to prove the resonance entrainment ability of (13) due to the filter (5) use (this simplest filter does not guarantee an accurate differentiation without a steady-state error) and the control (3) application for the modes $\mathcal{J}$ cancellation (this control ensures the cancellation asymptotically, thus in a generic case the frequency spectrum of the multi-DOF system (8) for all $t \geq 0$ contains some modes from $\mathcal{J}$ ). These problems can be resolved using the control (4) instead of (3), and the nonlinear differentiator (6) instead of (5).

Theorem 1. Let Assumption 1 be satisfied and a constant $U>0$ be given. Then the control

$$
\begin{gathered}
u=R^{-1} v, v=\left[v_{1}, \ldots, v_{n}\right]^{T}, \\
v_{k}=U \operatorname{sign}\left(\hat{\dot{z}}_{k}\right), \\
v_{j}=-\beta \operatorname{sign}\left(\hat{\dot{z}}_{j}\right)-(\beta+\varepsilon) \operatorname{sign}\left(\kappa_{j} \hat{\dot{z}}_{j} / \omega_{j}^{2}+z_{j}\right), j \in \mathcal{J} ;
\end{gathered}
$$

where $2 \beta+\varepsilon=U$ and

$$
\begin{aligned}
& \hat{\dot{z}}_{i}=\zeta_{2, i} ; \\
\dot{\zeta}_{1, i}= & -\alpha \sqrt{\left|\zeta_{1, i}-z_{i}(t)\right|} \operatorname{sign}\left[\zeta_{1, i}-z_{i}(t)\right]+\zeta_{2, i},(15) \\
\dot{\zeta}_{2, i}= & -\rho \operatorname{sign}\left[\zeta_{1, i}-z_{i}(t)\right]-\chi \operatorname{sign}\left(\zeta_{2, i}\right)-\zeta_{2, i}, \\
& \zeta_{1, i}(0)=z_{i}(0), \zeta_{2, i}(0)=0, \quad i=\overline{1, n},
\end{aligned}
$$

with the parameters $\alpha, \rho$ and $\chi$ calculated in accordance with (7) for

$$
\begin{gathered}
L_{1}=\sqrt{\epsilon /(\eta \nu)} U, L_{2}=\left(\kappa+\omega^{2}\right) L_{1}+U, \\
\eta=\min \left\{0.5,0.25 \omega^{2}\right\}, \nu=\kappa \min \left\{\frac{1}{3}, \frac{0.5}{1+\omega^{-2} \kappa^{2}}\right\}, \\
\epsilon=0.25 \kappa \omega^{-2}+\kappa^{-1}
\end{gathered}
$$

ensures for the system (8) boundedness of solutions and the natural oscillation entrainment for all initial conditions in a finite time.

In this theorem the upper limit of the control $U$ is introduced for $v$ for brevity of formulation (it can be recalculated from an upper bound of the control $u$ ).

\section{B. Neural circuit interpretation}

The obtained controls (13), (14) have the structure of an artificial dynamical neural network conventionally used for CPG modeling.

The scheme of the control (13) is shown in Fig. 1 (the control (14) has a similar scheme). The input transformation $z=$ $P^{-1} C^{-1} y$ and the output multiplicative gain $R^{-1} \operatorname{diag}[U]$ can be interpreted as a linear static feedforward layer of an artificial neural network. The nonlinear and dynamical transformations, presented in the second layer in Fig. 1, can be considered separately or united in the single layer. As it is proven in [1], [7], [4], being adjusted in accordance with the method described in this work, the network ensures entrainment of the system (8) to the resonance oscillations.

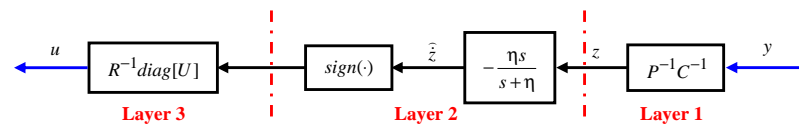

Figure 1. Scheme of the control (13)

The CPG based control scheme from the paper [10] is shown in Fig. 2, where $G$ and $H$ are some input and output transformation matrices, and CPG is modeled by a recurrent (feedback) network of dynamical neurons. The structure scheme of a neuron is given in Fig 3, where $v$ and $w$ are the scalar output and input of the neuron respectively, $\psi: \mathbb{R} \rightarrow \mathbb{R}$ is a sigmoidal activation function [10], the frequency $\omega_{o}$ represents the time lag and adaptation effects of the cell membrane and synapse modeled by the band pass filter. In the papers [10], [11] the ability of a network of such neurons for resonance entrainment is analyzed applying the HB method. The peculiarity of HB approach is that it is an approximate technique.

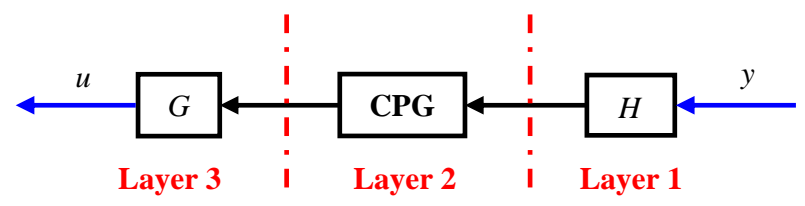

Figure 2. Scheme of the control from [10]

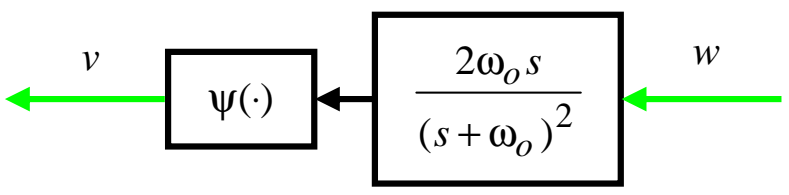

Figure 3. Scheme of an artificial neuron from [10]

Thus the schemes from Figs. 1 and 2 have the same number of layers. The first and the third layers have the same structure (the constant weights). The intermediate second layers are also similar; in Fig. 1 this layer is modeled by a parallel connection of neurons presented in Fig. 3 for $\psi(\cdot)=\operatorname{sign}(\cdot)$ with a low pass filter. 
Therefore, based on the entrainment framework founded in [10], [11], this paper presents an alternative methodology for neuronal circuit design for resonance entrainment of (8). The new approach has rigorous theoretical background for choice of the parameters. The proposed model of neural network is simpler than conventionally used, but it assumes a kind of centralized information processing (the controls proposed in [10], [11] are decentralized). The necessity of centralized information processing follows optimality of the control (13), i.e. to ensure an optimal performance we have to take care about the whole system (decentralized optimization of subsystems may not lead to the optimality of the integral system). This optimality of the control (13) that minimizes of the control energy consumption for resonance entrainment could be important for robotic system design.

\section{ApPLICATION}

Let us take the linear part of the example from [10]:

$$
J=\frac{1}{12} I_{3}, B=\left[\begin{array}{ccc}
1 & -1 & 0 \\
0 & 1 & -1 \\
0 & 0 & 1
\end{array}\right], C=B^{T}, K=B C
$$

where $I_{3}$ is the identity matrix with dimension $3 \times 3, \varepsilon=0.1$ and $\eta=10$. This system has natural frequencies:

$$
\omega=\left[\begin{array}{lll}
6.24 & 4.32 & 1.54
\end{array}\right]^{T} .
$$

In Fig. 4 the results of the control (13) application is shown where

$$
U= \begin{cases}U_{1} & \text { if } t \leq 10 \\ U_{2} & \text { if } t>10\end{cases}
$$

$U_{1}=\left[\begin{array}{lll}-5 & -1 & 0.1\end{array}\right]^{T}$ and $U_{2}=\left[\begin{array}{lll}5 & -1 & -0.1\end{array}\right]^{T}$. Thus for $t \leq 10$ the system oscillate with the frequency $\omega_{3}=1.54$, next for $t>10$ the control (13) in a finite time activates the natural oscillations with $\omega_{1}=6.24$. Such a change of oscillation frequency (or a normal mode) corresponds to a transition from the "walking" mode to the "running" one in animals.

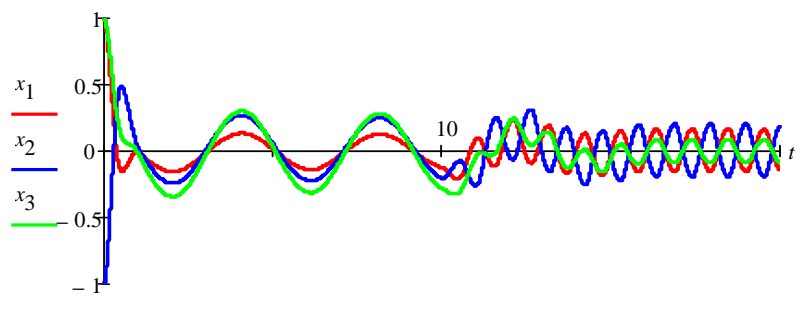

Figure 4. The energy-optimal control

The results of the control (13) application confirm its entrainment abilities to the resonance oscillations at natural frequencies. The switching between different frequencies (mode of oscillation) can be carried out by the incoming parameter setup ( $U$ or $E_{d}$ ).

\section{CONCLUSIONS}

The entrainment mechanism to a resonance mode based on CPG from [10], [11] is extended. The energy-optimal control [7], [8] is used to design the entrainment neural networks. The obtained conditions of oscillation at a natural frequency are based on Lyapunov analysis and the optimal control theory (the harmonic balance method is avoided). It is shown, that the resonance oscillations can be reached in a finite time. The proposed equations of neural network are simpler than used in [10], [11]. The results of computer simulation demonstrate good entrainment ability of the proposed approach.

Acknowledgments: The third author gratefully acknowledges the support from the National Science Foundation under No.0654070 and No.1068997, and by the Office of Naval Research, under MURI Grant N00014-08-1-0642.

\section{REFERENCES}

[1] Andrievsky B.R. Computation of the excitability index for linear oscillators. Proc. ECC-CDC 2005, Seville, Spain, 2005.

[2] Delcomyn F. Neural basis of rhythmic behavior in animals. Science, 210, 1980.

[3] Efimov D.V., Fradkov A.L. Hybrid adaptive resonance control using adaptive observers for vibration machines. Proc. ECC 2007, Kos, Greece, 2007, 2627-2634.

[4] Efimov D.V., Fradkov A.L. Hybrid adaptive resonance control using speed gradient approach for vibration machines. Proc. ACC 2007, New-York, USA, 2007.

[5] Efimov D., Fradkov A.L. Wave Control in the Lattices of Linear Oscillators. Proc. IEEE CDC 2010, Atlanta, 2010.

[6] Efimov D.V., Fridman L. A Hybrid Robust Non-Homogeneous FiniteTime Differentiator. IEEE Trans. Automatic Control, 56(5), 2011, pp. 1213-1219.

[7] Fradkov A.L. Exploring nonlinearity by feedback. Physica D, 128, 1999, pp.159-168.

[8] Fradkov A.L. Feedback Resonance in Nonlinear Oscillators. Proc. 5th European Contr.Conf., Karlsruhe, 1999.

[9] Fradkov A.L. Cybernetical Physics: From Control of Chaos to Quantum Control. Springer, 2007.

[10] Futakata Y., Iwasaki T. Entrainment to natural oscillations via uncoupled central pattern generators. IEEE Trans. Automatic Control, 56(5), 2011, 1075-1089.

[11] Futakata Y., Iwasaki T. Formal analysis of resonance entrainment by central pattern generator. Journal of Mathematical Biology, 57(2), 2008, 183-207.

[12] Desch W., Logemann H., Ryan E.P., Sontag E.D. Meagre functions and asymptotic behaviour of dynamical systems. Nonlinear Anal., 44(8, Ser. A: Theory Methods), 2001, pp. 1087-1109.

[13] Geering H.P. Optimal Control with Engineering Applications. Springer, 2007.

[14] Grillner S., J.T. Buchanan, P. Walker, Brodin L. Neuronal Control of Rhythmic Movements in Vertebrates. New York: Wiley, 1988.

[15] Holt K.G., Hamill J., Anders R.O. Predicting the minimal energy costs of human walking. Medicine and Science in Sport and Exercise, 23(4), 1991, 491-498.

[16] Orlovsky G.N., T.G. Deliagina, Grillner S. Neuronal Control of Locomotion: From Mollusc to Man. Oxford University Press, 1999. 